\title{
Algorithms and Machine Learning
}

\section{Manu Mitra}

Department of Electrical Engineering, Alumnus of University of Bridgeport, Bridgeport, United States. mmitra@my.bridgeport.edu

\section{INTRODUCTION}

Machine learning is a model that learns patterns in data and then calculates similar patterns in new data. For instance, if you want to categorize children's books, it would mean that instead of setting up exact instructions for what establishes a children's books, experts can set the computer hundreds of examples of children's books. Then the computer find the pattern in that books and uses that pattern to recognize future books in that particular category. However, Machine Learning is actually a subset of artificial intelligence that assists computers to learn without explicitly programmed with predefined instructions. It concentrates on development of computer applications that can teach themselves to develop and change when open to new data. This calculation ability, in addition to the computer's ability to process massive amounts of data; it supports machine learning to handle complex business conditions with efficiency and preciseness. [1]

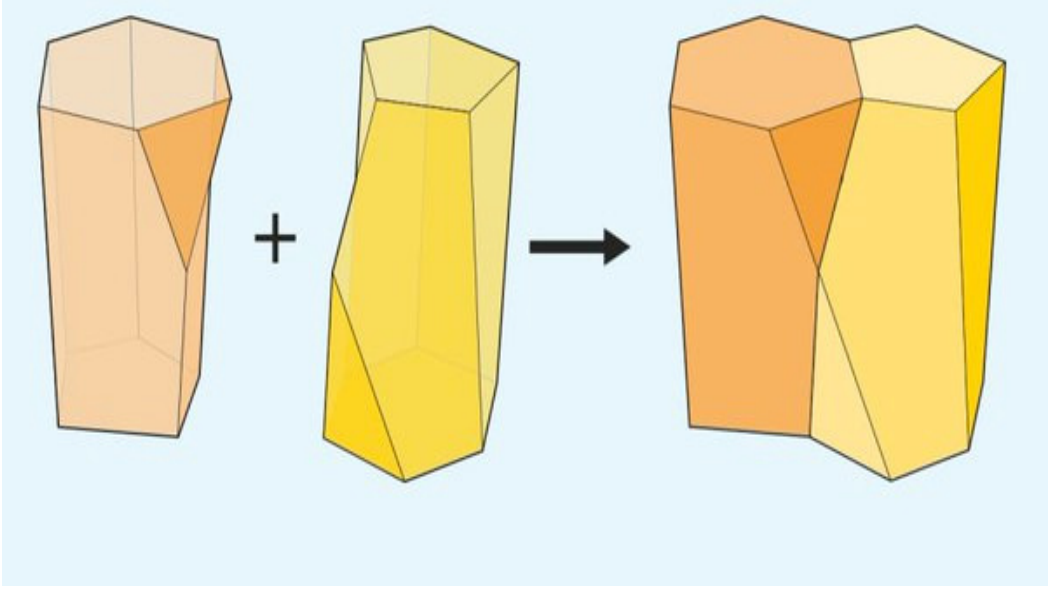

Fig1. Illustrates machine learning pattern. Image Credit: Richa Bhatia [1]

\section{MACHINE LEARNING IN INFORMATION THEORY}

The basic idea goes something like this: because of the ability to ignore useless information, a class of machine learning algorithms called deep neural networks can learn general concepts from raw data. For instance, recognizing cats after encountering tens of thousands of images of various cats in different variations. Early layers encode the "cat" label along with all the raw information required for calculation. Succeeding layers then compress the information as if through a bottleneck. Unnecessary data, like color of the cat's coat, saucer of milk nearby, are disregarded; leaving only general structures behind. Information theory offers bounds on how optimal layer is, in terms of how well it can balance the competing demands of compression and calculation. While the concept of compressing inputs may still play a vital role in machine learning. [2, 3] 


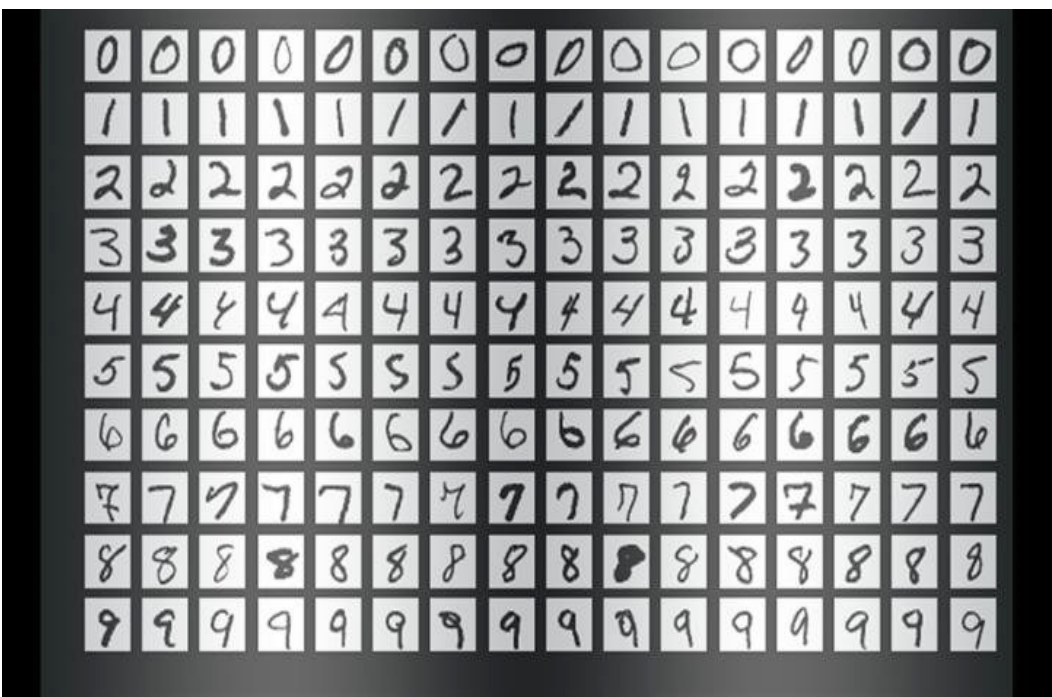

Fig2. Illustrates examples from the MNIST handwritten digits database. Image Credit: Josef Steppan [3]

\section{Machine Learning Algorithim to Track Cosmic Radio}

Fast radio burst are bright pulses of radio discharge mere of milliseconds in time interval, it was thought to originate from distant galaxies. The source of these discharges are unclear. Although, there are theories that range from highly magnetized neutron blasted by gas creeks from neighbor supermassive black hole. The burst properties are consistent with the signatures of technology created by an advanced civilization. For successful machine learning algorithm to search new types of signals that could be coming from extraterrestrial civilizations. While most fast radio bursts are one-offs, FRB 121102 is unique in emitting repeated bursts. This performance has drawn attention of many astronomers expecting to pin down the cause and the extreme physics involved in fast radio bursts. Artificial Intelligence algorithm dredged up the radio signals from the data were recorded over a five-hour of time interval on Aug 26, 2017 by Green Bank Telescope in West Virginia. An earlier examination of 400 terabytes of data employed standard computer algorithms to recognize 21 bursts during that interval of time. $[4,5]$

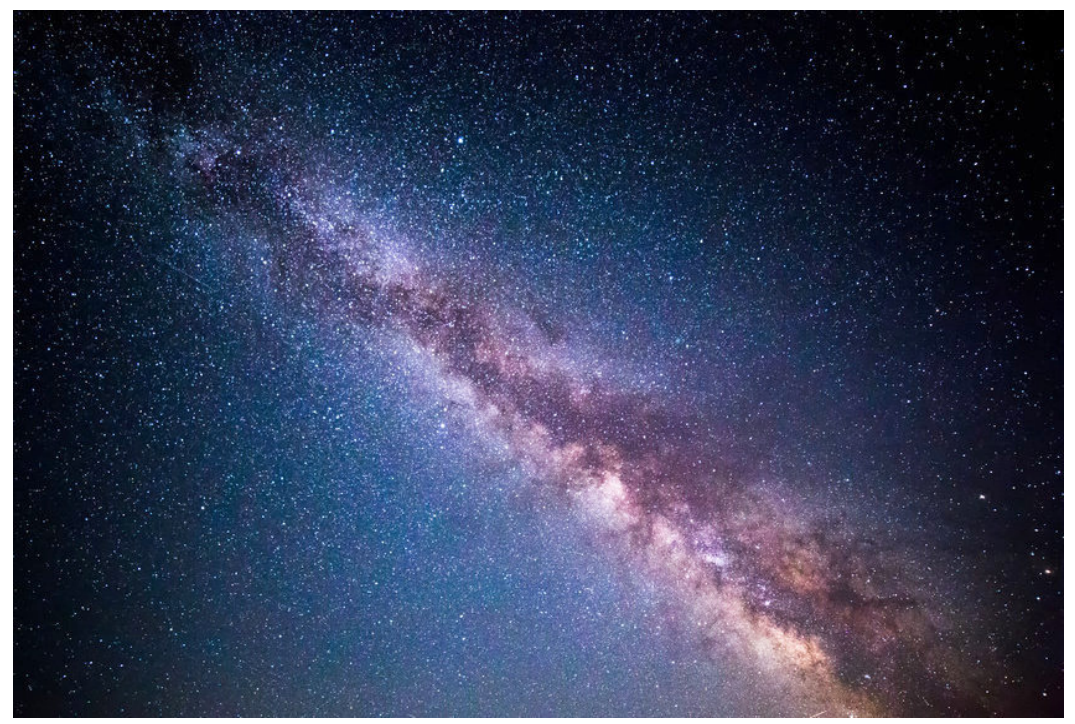

Fig3. IllustratesMilky Way (stock image). Image Credit: mandritoiu/Fotolia [5] 


\section{Algorithim to CAlculate Led Materials}

Scientists from University of Houston devised a new machine learning algorithm that is capable enough to run on a personal computer and calculate the prediction properties of more than 100,000 compounds in search of those most likely to be effective phosphors for Light Emitting Diode (LED) lighting. Scientist used machine learning to scan large number of compounds for key attributes including Debye temperature and chemical compatibility. Brgoch previously verified that Debye temperature is correlated with efficiency. LED based bulbs work by using small amounts of rare earth elements usually europium or cerium, replaced within a ceramic or oxide host; the interaction between the two materials decides the performance. This task started with a list of 118, 287 possible inorganic phosphor compounds from Pearson's Crystal Structure Database; the algorithm carved just over 2,000. Another 30 seconds and it produced a list of about two dozen favorable materials. It proved to be stable, with a quantum yield of efficiency of 95 percent. $[6,7]$

\section{MACHINE LEARNiNG TO IDENTIFY BRAIN TUMORS}

Brain tumors include a wide range of tumors depending on the cell type, the aggressiveness and stage of tumor. Speedy and precisely characterizing the tumor is a critical part of treatment planning. It is a duty currently allocated for trained radiologist but in future computing with high performance computing, it will play a very vital role in supporting and assisting. More than a dozen students and researchers were given in advance with 300 sets of brain images on which all teams standardized their methods. In the final part of the task, they were provided data from 140 patients and had to recognize the location of tumors and segment them into various tissue types over the time of only two days. Image analysis, processing and calculating the data that researcher's team used had two major steps: a supervised machine learning step where the computer creates a probability map for the target classes; and second was to combine these probabilities with biophysical model that signifies how tumors develop in mathematical terms which enforces limits on the analyses and assists to find correlations. With 1.5 million voxels per brain and 300 brains to assess, that means computer have to search at half billion voxels for every new voxel of the 140 unknown brains that it analyzes, determining for each whether the voxel signifies a tumor or healthy tissue. [8]

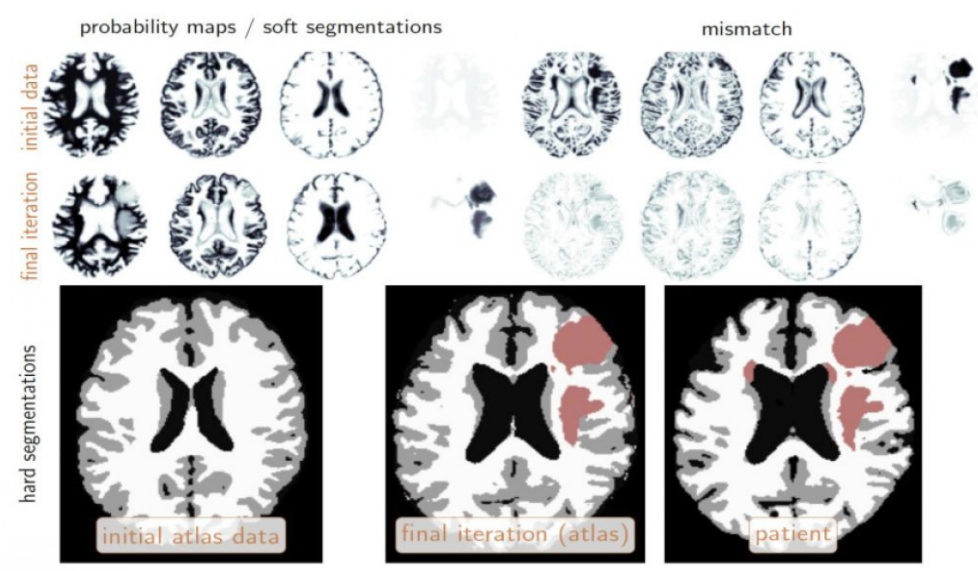

Fig4. Illustratestop row shows the initial configuration. The second row shows the same configuration at the final iteration of our coupled tumor inversion and registration scheme. The three images on the bottom show the corresponding hard segmentation. The obtained atlas based segmentation (middle image) and the ground truth segmentation for the patient are very similar. Image Credit: Andreas Mang, Sameer Tharakan, Amir Gholami, Naveen Himthani, Shashank Subramanian, James Levitt, MuneezaAzmat, KlaudiusScheufele, Miriam Mehl, Christos Davatzikos, Bill Barth and George Biros [8] 


\section{Machine Learning in Condensed MatTer Physics}

A machine learning algorithm was designed to teach computers how to identify photos, speech patterns and hand written digits. It has now applied vastly different set of data; recognizing phase transitions between states of matter. Using gigabytes of data demonstrating different state configurations developed using simulation software on supercomputers. Researchers created a large collection of "images" to introduce into the machine learning algorithm. The result was it was able to distinguished phases of a simple magnet and could distinguish an ordered ferromagnetic phase from disordered high temperature phase. It was even able to locate the boundary or phase transition between phases. This shows that applying machine learning to condensed matter and statistical physics can open entirely new opportunities for research and gradually in real time application. $[9,10]$

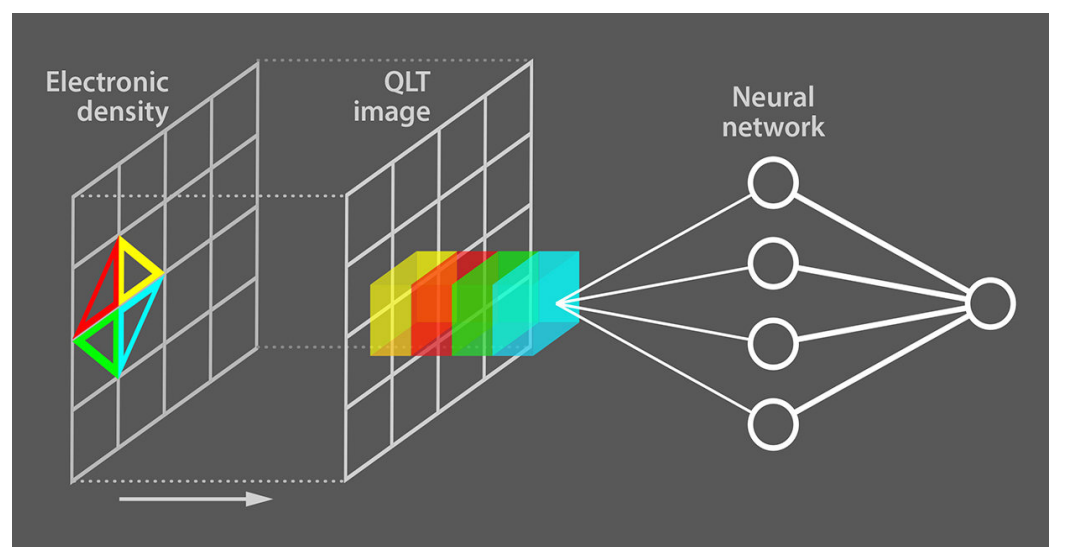

Fig5. Illustrates an exotic topological phase of matter was identified with a machine-learning approach. The left schematic illustrates a snapshot of the electronic density of the system. Using a quantum loop topography (QLT) technique, the neighboring triangular regions in the electronic density profile are translated to multidimensional images of the material's structure. These images show different insulating phases that are then fed into a neural network. (The four vertical circles are a hidden layer in the neural network.) The machine learns by example if the phase is topological or not. For future applications, the "educated" machine can detect topological phases on its own Image Credit: US Department of Energy [11]

\section{REFERENCES}

1. Bhatia, R. (2019, September 10). How Do Machine Learning Algorithms Differ From Traditional Algorithms? Retrieved from https://www.analyticsindiamag.com/how-do-machine-learning-algorithms-differ-fromtraditional-algorithms/

2. ArtemyKolchinsky, Brendan D. Tracey, Steven Van Kuyk. Caveats for information bottleneck in deterministic scenarios. Submitted to arXiv, 2019

3. Santa Fe Institute. (2019, January 24). Information theory holds surprises for machine learning. ScienceDaily. Retrieved March 8, 2019 from www.sciencedaily.com/releases/2019/01/190124095106.htm

4. Yunfan Gerry Zhang, Vishal Gajjar, Griffin Foster, Andrew Siemion, James Cordes, Casey Law, Yu Wang. Fast Radio Burst 121102 Pulse Detection and Periodicity: A Machine Learning Approach. arXiv.org, 2018

5. University of California - Berkeley. (2018, September 10). Artificial intelligence helps track down mysterious cosmic radio bursts: Machine learning algorithm also helps search for new kinds of signals from extraterrestrial intelligence. ScienceDaily. Retrieved March 8, 2019 from www.sciencedaily.com/ releases/2018/09/180910160509.htm 
Algorithms and Machine Learning

6. YaZhuo, Aria Mansouri Tehrani, Anton O. Oliynyk, Anna C. Duke, JakoahBrgoch. Identifying an efficient, thermally robust inorganic phosphor host via machine learning. Nature Communications, 2018; 9 (1) DOI: 10.1038/s41467-018-06625-z

7. University of Houston. (2018, October 22). New algorithm can more quickly predict LED materials: Researchers report machine learning speeds discovery of new materials. ScienceDaily. Retrieved March 8, 2019 from www.sciencedaily.com/releases/2018/10/181022085832.htm

8. University of Texas at Austin, Texas Advanced Computing Center. (2017, October 5). Scientists enlist supercomputers, machine learning to automatically identify brain tumors. ScienceDaily. Retrieved March 8, 2019 from www.sciencedaily.com/releases/2017/10/171005141736.htm

9. Juan Carrasquilla, Roger G. Melko. Machine learning phases of matter. Nature Physics, 2017; DOI: 10.1038/ nphys 4035

10. Perimeter Institute for Theoretical Physics. (2017, February 13). Researchers apply machine learning to condensed matter physics. ScienceDaily. Retrieved March 7, 2019 from www.sciencedaily.com/ releases/2017/02/170213171444.htm

11. Physics/US Department of Energy. (2018, January 8). Machine learning and neural networks recognize exotic insulating phases in quantum materials. Retrieved from https:/phys.org/news/2018-01-machineneural-networks-exotic-insulating.html

Citation: Manu Mitra, "Algorithms and Machine Learning". American Research Journal of Electronics and Communication Engineering; vol 1, no. 1; pp: 1-5.

Copyright (c) Manu Mitra. This is an open access article distributed under the Creative Commons Attribution License, which permits unrestricted use, distribution, and reproduction in any medium, provided the original work is properly cited. 